\title{
A High-Frequency Intensity Difference Location Algorithm in Three Dimensions Based on Helmet- Mounted Microphone Array
}

\author{
Y. Zhang, B.B. Shen \\ Information Engineering College \\ University of Dalian \\ Dalian, China
}

\begin{abstract}
The common localization algorithms of microphone array is based on linear array or circle array which is unblocked (there is no block between sound source and microphone). This paper we research the sound localization algorithm based on helmet-mounted microphone array which is a kind of blocked array in the three-dimensional space. This paper takes the attenuation of high frequency when passing through by helmet into account, and utilizes intensity decay of different backward microphones to construct localization algorithm. Experimental results show that the localization method of the high frequency sound signal based on the direct path improves the accuracy of localization compared with common algorithm based on unblocked microphone array.
\end{abstract}

Keywords-helmet-mounted microphone array; intensity difference; sound localization; three-dimensional

\section{INTRODUCTION}

The sound source localization has been widely used in various fields, such as hearing aids, robot, video conference, etc. $[1,2]$. All of these applications utlize microphone arrays to localize sound sources. For example, robot uses the microphone array installed on its head to obtain speaker's position, so as to steer the video camera towards controller. In battlefield, soldiers obtain snipers' locations with microphone arrays mounted on their heads, to guide counterforce.

Currently, sound source localization algorithms can be divided into three types according to different parameters: the time difference $[3,4]$, the room impulse response difference and the microphone channel difference. These methods generally use unblocked microphone arrays (where is no obstacle between microphone and sound source), and can improve the positioning accuracy by increasing the number of microphones.

We need to design a more simple and precise microphone array in order to achieve better positioning requirements. Thus, various forms of the microphone array are constantly being developed, such as helmet-mounted microphone array. Hyung O. Park and Alireza A. Dibazar simplify the number of microphones on the helmet-mounted microphone array and improve the positioning speed [5]. Flaviu Ilie Bob designed a portable and low-cost positioning system for the purpose of obtains a more general application [6]. Of course, accurate analysis of the intensity of the sound signal is more useful to sound source localization. Therefore, Hüseyin Hacihabiboğlu designed an open-sphere 3-D intensity probe which set to determine the three-dimensional sound intensity [7, 8], and it's a better system to measure the intensity of sound signal. These results of localization research will have a profound impact on the study of the microphone array.

In this paper, we made a further study on the localization based on helmet-mounted microphone array by the high frequency speech signal. Utilizing the signal intensity attenuation of the backward microphones on the helmet, we propose a localization algorithm based on the high frequency intensity difference of helmet-mounted microphone array in the three-dimensional space. The results show that the localization algorithm based on high frequency intensity difference improves the localization accuracy. And the search algorithm greatly reduces the amount of calculation; therefore, it is convenient to be used in real-time localization system. And the search algorithms can locate the position of any sound source in the three-dimensional space.

\section{HELMET-MOUNTED MICROPHONE ARRAY}

At present, the commonly used microphone arrays are unblocked so that the acoustic signal directly propagates to each array element. This paper researches the localization algorithm based on the helmet mounted microphone array [9], which is a kind of blocked array. Microphones are mounted on a helmet of a dummy, and its structure diagram as shown in Figure 1.
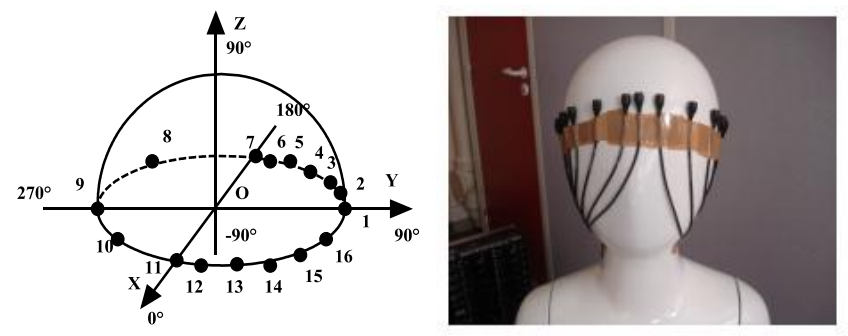

(a) Structure diagram. (b) Helmet-mounted microphone array

FIGURE I. ST RUCT URE DIAGRAM AND PLANE OF A HELMETMOUNTED MICROPHONEARRAY

For speech signals of different frequencies, which reaches the microphone path is different, because of the helmet mounted microphone array is a blocked array. For high frequency signal, the intensity of signal is attenuated, and lowfrequency signal occur diffraction. In this paper, we focus on 
the high-frequency signal (which frequency is greater than $5000 \mathrm{~Hz}$ ) propagation path and according the intensity attenuation between forward microphone (microphones and sound source on the same side of helmet) and backward microphone (microphones and sound source on the different side of helmet) to construct localization algorithm.

Sound source signal straight to reach the forward microphones, and for the backward microphones, the high frequency speech signal also straight reach, the propagation path show in Figure 2. However, when passing through the helmet, the intensity of the speech signal will be attenuated; the helmet attenuated the high-frequency signal intensity that is proportional to the penetration distance. $S$ is the location of the sound source, $M$ is the microphone on the helmet and it's coordinate is $(a, b, 0) \cdot S M$ is the propagation path of the high-frequency speech signal.

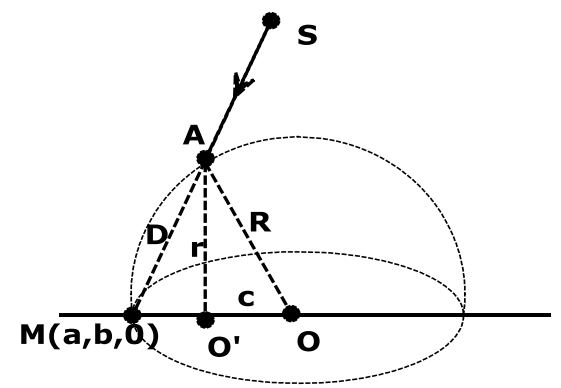

FIGURE II. HELMET MICROPHONE ARRAY PROPAGATIONPATH OF HIGH-FREQUENCY SOUND SIGNAL

\section{HIGH-FREQUENCY SOUND SOURCE LOCALIZATION METHOD IN THREE-DIMENSIONAL SPACE}

We suppose $s(t)$ (the sound source signal) is the signal received by the forward microphones of the helmet and $x_{i}(t)$ is the signal received by the backward microphones of the helmet. So that we can draw penetration distance $D_{i}$ by the formula $x_{i}(t)=s(t) / D_{i}+\xi_{i}(t)$, where $\xi_{i}(t)$ is additive white Gaussian Noise. Here we only take the attenuation caused by penetration distance in helmet into accounted, and neglect the attenuation caused by air. Measuring the intensity of microphone $M$ is $x_{M}(t)$, and then we can draw penetration distance $D$. In this sphere of the helmet, the point which is away from microphone $M$ is $D$ form a circle which center is point $O^{\prime}$. It becoming a cone surface when the circle gradually to a three dimensional expansion, and the vertex of the cone surface is the microphone $M$.

In order to obtain the equation of the cone surface, we set the radius $O^{\prime} A$ of the circle $O^{\prime}$ is $r$, the distance between $O^{\prime}$ and $O$ is $c$, and the radius $O A$ of the sphere of the helmet is $R$. From Figure 2, we can draw

$$
\left\{\begin{array} { l } 
{ D ^ { 2 } = r ^ { 2 } + ( R - c ) ^ { 2 } } \\
{ R ^ { 2 } = r ^ { 2 } + c ^ { 2 } }
\end{array} \Rightarrow \left\{\begin{array}{l}
r=\sqrt{R^{2}-c^{2}} \\
c=\frac{2 R^{2}-D^{2}}{2 R}
\end{array}\right.\right.
$$

Then, we set the vector $\vec{n}$ perpendicular to the plane of the circle $O^{\prime}$ is $(a, b, 0)$, the vector $\vec{u}$ in the round face is $(b,-a, 0)$, and the coordinate of $O^{\prime}$ is

$$
\begin{aligned}
& O^{\prime}\left(c_{x}, c_{y}, c_{z}\right)=\left(a \frac{c}{R}, b \frac{c}{R}, 0\right) \\
& \vec{v}=\vec{n} \times \vec{u}=\left(0,0, \frac{-a^{2}-b^{2}}{a^{2}+b^{2}}\right)=(0,0,-1)
\end{aligned}
$$

Unitization vector $\vec{u}$ and $\vec{v}$,

$$
\left\{\begin{array}{l}
\tilde{u}=\frac{\vec{u}}{\|\vec{u}\|}=\left(\frac{b}{R},-\frac{a}{R}, 0\right) \\
\tilde{v}=\frac{\vec{v}}{\|\vec{v}\|}=(0,0,-1)
\end{array}\right.
$$

So we can conclude the parametric equation of the circle in space,

$$
\left\{\begin{array}{l}
x=c_{x}+r\left(\tilde{u}_{x} \cos t+\tilde{v}_{x} \sin t\right)=a \frac{c}{R}+r \frac{b}{R} \cos t \\
y=c_{y}+r\left(\tilde{u}_{y} \cos t+\tilde{v}_{y} \sin t\right)=b \frac{c}{R}-r \frac{a}{R} \cos t \\
z=c_{z}+r\left(\tilde{u}_{z} \cos t+\tilde{v}_{z} \sin t\right)=-r \sin t
\end{array}\right.
$$

Then we can write the parametric equation of cone surface by circle equation,

$$
\left\{\begin{array}{l}
\bar{x}=x_{0}+s x=a+s\left(a \frac{c}{R}+r \frac{b}{R} \cos t\right) \\
\bar{y}=y_{0}+s y=b+s\left(b \frac{c}{R}-r \frac{a}{R} \cos t\right) \\
\bar{z}=z_{0}+s z=-s r \sin t
\end{array}\right.
$$

where $s$ is a scaling factor in the equation (6).

Through the above derivation, we can draw a different cone surface equation by another backward microphone. Three cone surface equations can be drawn if we select three different microphones, which shown in Figure 3. These three cone surface intersect at one point, this point is the sound source location which we want to search.

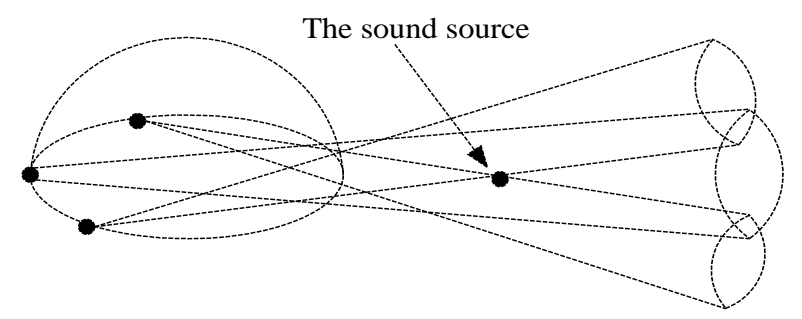

FIGURE III. THE RESULT OF HIGH-FREQUENCY SOUND SOURCE LOCALIZATION BYTHREE MICROPHONES 


\section{EXPERIMENTAL RESULT S}

\section{A. Experimental Data Configuration}

In a $6 m \times 4 m \times 2 m$ room, the microphone array on the dummy's head is shown in Figure.1. Sixteen microphones are installed on a helmet of radius $R=0.09 \mathrm{~m}$, the central angles of microphones between 1 to 7 and 11 to 16 are $\pi / 12 \mathrm{rad}$, and the central angles between the remaining microphones are $\pi / 4 \mathrm{rad}$.

In this paper, the algorithm process is as follows: First, the sound source signal is filtered by a high-pass filter whose passband cutoff frequency is $5400 \mathrm{~Hz}$, cutoff band starting frequency is $5000 \mathrm{~Hz}$, pass-band maximu m attenuation is $3 \mathrm{~dB}$, and stop-band minimum attenuation is $50 \mathrm{~dB}$, high frequency signal obtained after filtering. Second, select three microphones from the backward microphones, measuring the sound signal intensity received by each of the three microphones, thereby to obtain the corresponding penetration distance $D_{i}$. Third, according to the formula (6), we can draw a cone surface from each mic rophone, and then three cone surfaces can be drawn by three backward microphones. The result obtained is shown in Figure 3. The common intersection of three cone surfaces is the location of the sound source.

\section{B. Experimental Results}

As illustrated in Figure 4, it's draw the relationship between the average error and azimuth of the sound source (we set the sound source position of the helmet at a distance of $3 \mathrm{~m}$, the horizontal angle of the sound source is $270^{\circ}$ which shown in Figure 1(a), and the azimuth of the sound source moves from $90^{\circ}$ to $90^{\circ}$ every $10^{\circ}$, measure five times and take the average of errors at each position). From it can be seen that the average error value (the average error value of distance between the real sound source and the measurement of sound source) is smaller when the azimuth is close to $0^{\circ}$. It's mean that the localization will be more accurate when the sound source and the helmetmounted microphone at a same plane. However, the sound source position error is not large more when the azimuth is not $0^{\circ}$. This shows that this algorithm is suitable for threedimensional positioning.

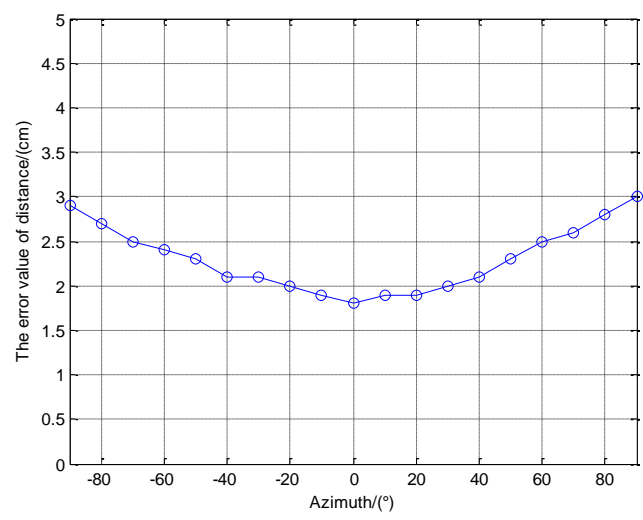

FIGURE IV.THE RELATIONSHIP BETWEEN THE AVERAGE ERROR VALUE AND AZIMUTH

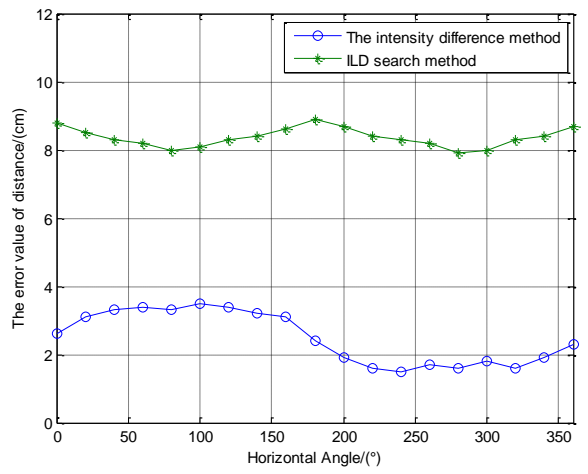

\section{FIGURE V. COMPARISON OFTWO MET HODS POSIT IONING AVERAGE ERROR VALUE}

Two curves in Figure 5 represent the average error value with two different search methods (we set the distance from the microphone to the sound source is always $3 \mathrm{~m}$, the azimuth of sound source is $0^{\circ}$ and the horizontal angle changed from $0^{\circ}$ to $360^{\circ}$ every $20^{\circ}$ ). The upper one uses the ILD search method [10], and the lower one is this paper's method which uses the intensity difference to search sound source in the threedimensional space. We can clearly see that the average error value of the intensity difference method always less than the ILD search method. Through the comparison we can obtain that the method of high frequency intensity difference location based on helmet-mounted microphone array greatly improve the positioning accuracy.

\section{CONCLUSION}

In this paper, the localization algorith $\mathrm{m}$ based on a helmetmounted microphone array utilizes the direct path of high frequency band to construct the location framework, and uses the method of high frequency intensity difference to search for the location of a sound source in the three-dimensional space. The results of simulation show that the localization algorithm based on the direct path of high-frequency sound signal in this paper reduces the amount of calculation in the same noise, effectively and greatly improves the positioning accuracy.

\section{ACKNOWLEDGEMENT S}

This project is supported by the National Natural Science Foundation of China (No. 61201420).

\section{REFERENCES}

[1] Kai Wang, Zhiya Zong, Xiaowei Sun, and Weiren Shi, Study on improved adaptive eigenvalue decomposition algorithm for acoustic source localization[J].Chinese Journal of Scientific Instrument, 2013,34(6): 1241-1246.

[2] Tiete J,Domínguez F,Silva Bd,Segers L,Steenhaut K, and Touhafi A, Sound Compass: A Distributed MEMS Microphone Array-Based Sensor for Sound Source Localization[J].Sensors (Basel), 2014,14(2): 1918-1949.

[3] Sheng Zhao, Mufan Cui,Lei You and Hongpeng Wang,Sound source localization technology based on small microphone array [J].J.Huazhong Univ. Of Sci.\& Tech.(Nat ural Science Edition), 2013,41(1):188-191.

[4] Yin F, Wang S, and Chen Z, A Time Delay Estimation Method Based on Wavelet Transform and Speech Envelope for Distributed Microphone arrays[J].2013,13(3):39-44.

[5] Hyung O. Park, Alireza A. Dibazar, and Theodore W. Berger, DESIGN OF A HELMET-MOUNTED MICROPHONE ARRAY FOR SOUND 
LOCALIZATION. ICASSP 2010, 1478-1481.

[6] Flaviu Ilie Bob, Embedded Solution for Universal Acoustic Source Distance Localization Using Three Microphones.ISET C 2012, 359-362.

[7] Hüseyin Hacihabiboğlu, Theoretical Analysis of Open Spherical Microphone Arrays for Acoust ic Intensity Measurements.TASLP 2014, $465-476$.

[8] Hüseyin Hachabiboğlu, ON THE ACCURACY OF OPEN SPHERICAL MICROPHONE ARRAYS FOR MEASURING ACOUSTIC INTENSITY.WASPAA 2013,1-4.

[9] Hyung-Ook Park,Alireza A. Dibazar,and Theodore W. Berger, DESIGN OF A HELMET-MOUNTED MICROPHONE ARRAY FOR SOUND LOCALIZATION[J].ICASSP 2010, 14-19.

[10] Stanley T. Birchfield and Rajitha Gangishetty, ACOUST IC LOCALIZATION BYINTERAURAL LEVEL DIFFERENCE. ICASSP2005. 